\title{
Chemotype classification and biomarker screening of male Eucommia ulmoides Oliv. flower core collections using UPLC- QTOF/MS-based non-targeted metabolomics
}

\author{
Panfeng Liu ${ }^{1,2,3}$, Lu Wang ${ }^{1,2,3}$, Qingxin Du ${ }^{1,2,3}$, Hongyan Du ${ }^{\text {Corresp. 1, 2,3 }}$ \\ ${ }^{1}$ Paulownia Research \& Development Center of China, National Forestry and Grassland Administration, Zhengzhou, Henan, China \\ 2 Key Laboratory of Non-timber Forest Germplasm Enhancement \& Utilization of State Forestry and Grassland Administration, Zhengzhou, Henan, China \\ 3 Non-timber Forestry Research \& Development Center, Chinese Academy of Forestry, Zhengzhou, Henan, China \\ Corresponding Author: Hongyan Du \\ Email address: dhy515@caf.ac.cn
}

Background: In Chinese health care industry, male Eucommia ulmoides Oliv. flowers are newly approved as a raw material of functional food. Core collections have been constructed from conserved germplasm resources based on phenotypic traits and molecular markers. However, little is known about these collections' phytochemical properties. This study explored the chemical composition of male E. ulmoides flowers, in order to provide guidance in the quality control, sustainable cultivation, and directional breeding of this tree species. Methods: We assessed the male flowers from 22 core collections using ultra-high-performance liquid chromatography and quadrupole time-offlight tandem mass spectrometry (UPLC-QTOF/MS) non-targeted metabolomics, and analyzed them using multivariate statistical methods including principal component analysis (PCA), hierarchical cluster analysis (HCA), and orthogonal partial least squares discriminant analysis (OPLS-DA). Results: We annotated a total of 451 and 325 metabolites in ESI+ and ESI- modes, respectively, by aligning the mass fragments of the secondary mass spectra with those in the database. Four chemotypes were well established using the ESI+ metabolomics data. Of the 29 screened biomarkers, 21, six, 19, and five markers corresponded to chemotypes I, II, III, and IV, respectively. More than half of the markers belonged to flavonoid and amino acid derivative classes. Conclusion: Nontargeted metabolomics is a suitable approach to the chemotype classification and biomarker screening of male $E$. ulmoides flower core collections. We first evaluated the metabolite profiles and compositional variations of male $E$. ulmoides flowers in representative core collections before establishing possible chemotypes and significant biomarkers denoting variations. We used genetic variations to infer the metabolite compositional variations of male $E$. ulmoides flower core collections instead of using the geographical origins of the germplasm resources. The newly proposed biomarkers 
sufficiently classified the chemotypes to be applied for germplasm resource evaluation. 
1 Chemotype classification and biomarker screening of

2 male Eucommia ulmoides Oliv. flower core collections

3 using UPLC-QTOF/MS-based non-targeted

4 metabolomics

5 Panfeng Liu ${ }^{1,2,3}$, Lu Wang 1,2,3, Qingxin Du ${ }^{1,2,3}$, Hongyan $\mathrm{Du}^{1,2,3}$

$6 \quad{ }^{1}$ Paulownia Research \& Development Center of China, National Forestry and Grassland

7 Administration, Zhengzhou, Henan, China

$8{ }^{2}$ Key Laboratory of Non-timber Forest Germplasm Enhancement \& Utilization of State Forestry

9 and Grassland Administration, Zhengzhou, Henan, China

$10{ }^{3}$ Non-timber Forestry Research \& Development Center, Chinese Academy of Forestry,

11 Zhengzhou, Henan, China

12 Corresponding author:

13 Hongyan $\mathrm{Du}^{1,2}$

14 Email address: dhy515@caf.ac.cn

\section{Abstract}

Background: In Chinese health care industry, male Eucommia ulmoides Oliv. flowers are newly approved as a raw material of functional food. Core collections have been constructed from conserved germplasm resources based on phenotypic traits and molecular markers. However, little is known about these collections' phytochemical properties. This study explored the chemical composition of male E. ulmoides flowers, in order to provide guidance in the quality control, sustainable cultivation, and directional breeding of this tree species.

Methods: We assessed the male flowers from 22 core collections using ultra-high-performance liquid chromatography and quadrupole time-of-flight tandem mass spectrometry (UPLCQTOF/MS) non-targeted metabolomics, and analyzed them using multivariate statistical methods including principal component analysis (PCA), hierarchical cluster analysis (HCA), and orthogonal partial least squares discriminant analysis (OPLS-DA).

Results: We annotated a total of 451 and 325 metabolites in ESI+ and ESI- modes, respectively, by aligning the mass fragments of the secondary mass spectra with those in the database. Four chemotypes were well established using the ESI+ metabolomics data. Of the 29 screened biomarkers, 21, six, 19, and five markers corresponded to chemotypes I, II, III, and IV, respectively. More than half of the markers belonged to flavonoid and amino acid derivative classes.

Conclusion: Non-targeted metabolomics is a suitable approach to the chemotype classification and biomarker screening of male E. ulmoides flower core collections. We first evaluated the metabolite profiles and compositional variations of male E. ulmoides flowers in representative core collections before establishing possible chemotypes and significant biomarkers denoting variations. We used genetic variations to infer the metabolite compositional variations of male $E$. 
resources. The newly proposed biomarkers sufficiently classified the chemotypes to be applied for germplasm resource evaluation.

\section{Introduction}

Eucommia ulmoides Oliv. is a multifunctional tree species endemic to China. Due to geological processes during the quaternary glaciation, this monotypic genus remained primarily in Mideastern China (Call \& Dilcher, 1997). After its introduction to other regions of China, $E$. ulmoides was planted in $350,000 \mathrm{hm}^{2}$ of land across 28 provincial administrative districts (Du et al., 2013). The bark of E. ulmoides has been used as a traditional herbal medicine for almost 2,000 years (Feng \& Liang, 1996).Currently, the tree is fully utilized as a part of the rubber modification, medicine, health food, pollution-free breeding and landscaping industries ( $D u$ et al., 2013; Du et al., 2015).

E. ulmoides is strictly dioecious and its male capitulum inflorescence consists of five to 11 clustered achlamydeous flowers. Several bractlets are attached to the tiny pedicel, and each male flower is composed of eight to 12 stamens. In the Yellow River basin and other parts of northern China, the male flower blooming period is between mid -March and early April and lasts approximately 20 days. This is eight to 15 days earlier than the bolooming period of the female flower (Liu, 2010; Liang, 2007). The natural compounds that have been extracted from male flowers, including geniposide, geniposidic acid, aucubin, chlorogenic acid, and flavonoids, have important anti-inflammatory, bacteriostatic, antihypertensive, antihyperlipidemic, hepatoprotective, choleretic, and cardiovascular protective qualities (Dong et al., 2005; Zhang et al., 2007). In 2014, the National Health Commission of China added male E. ulmoides flowers to their novel food list (National Health Commission, 2014).

The research and innovation team of the National Germplasm Resources Bank of Major Famous Tree Species in the North, located in Yuanyang, Henan Province, has conserved nearly 2,000 E. ulmoides germplasm resources, including more than 750 male collections, since 2009. Previous studies have evaluated the diversity of the morphological traits, amino acid content, and main active components of 193 male collections ( $D u$ et al., 2017a; Du et al., 2017b; Du et al., 2016), and 33 male core collections were screened by analysing the phenotypic and SSR molecular marker data (Li et al., 2018a; Li et al., 2018b). However, these studies did not explore how metabolic composition is used to classify male flower collections or determine the existence of reasonable chemical indicators when distinguishing traits, which are important to the quality formation and commercialization processes.

The metabolic composition and chemotype of different germplasm resources should be accurately evaluated to effectively expand genetic base, breeding innovation, and direct cultivation. Non-targeted metabolomics is an unbiased approach that comprehensively excavates the metabolic fingerprints of biological samples, despite having limited linear range and poor repeatability (Fan et al, 2017). Because of the particularly high performances in both mass spectrometry (MS) and tandem MS (MS/MS) modes, the combination of ultra-high-performance liquid chromatography (UPLC) separation and quadrupole time-of-flight tandem mass spectrometry (Q/TOF-MS) detection has shown absolute superiority in qualitative and 
79

80

81

82

83

84

85

86

87

88

89

90

91

92

93

94

95

96

97

98

99

100

101

102

103

104

105

106

107

108

109

110

111

112

113

114

115

116

117

118

quantitative compound analysis(Taamalli et al., 2014; Wolfender et al., 2008; Da Silva et al., 2017). Coupled with multivariate statistical methods, UPLC-Q/TOF-MS is an optimized compound characterization approach that has been used to analyse ginseng, Ficus deltoidea, Garcinia oblongifolia, and many traditional Chinese medicinal herbs (Zhu et al., 2019; Afzan et al., 2019; Ning et al., 2013; Yu et al., 2018).

In this study, we profiled the metabolite constituents of 22 fresh male E. ulmoides core collections flowers grown under similar environmental conditions and cultivation practices. We processed data using multivariate statistical methods, including principal component analysis (PCA), hierarchical cluster analysis (HCA), and orthogonal partial least squares discriminant analysis (OPLS-DA), to determine the possible phytochemical phenotypes and metabolomic markers across the collections. Our study provides a comprehensive view of $E$. ulmoides male flower metabolomes and a basis for the future utilization of germplasm evaluation, nutritional properties, and biological analysis.

\section{Materials \& Methods}

\section{Plant materials}

We sampled fresh male flowers in the full-bloom stage from 22 representative core collections in the germplasm pool in late March 2018. Collections were sampled from 13 provincial districts and conserved ex situ following grafting propagation in 2013. The male flower morphological characteristics and variations are provided in Table S1 and Fig.S1, respectively. For each collection, we randomly selected six biological replicates from two to four individuals, for a total of 132 independent samples for metabolite profiling. After removing bractlets and tiny leaves attached to the inflorescence, all samples were frozen immediately in liquid nitrogen and then stored at $-80^{\circ} \mathrm{C}$. Voucher specimens were deposited at the Key Laboratory of Non-timber Forest Germplasm Enhancement \& Utilization of State Forestry and Grassland Administration, Zhengzhou. The numbers and descriptions of the voucher specimens are contained in the raw data files (https://zenodo.org/record/3905465\#.Xw3Hjud5uUk).

\section{Extraction procedures}

We weighed each sample (ca. $15 \mathrm{mg}$ ), manually ground them into powder using liquid nitrogen, and processed all samples in one batch. The powdered samples were macerated with $120 \mu \mathrm{L}$ of precooled $50 \%$ methanol, mixed for $1 \mathrm{~min}$, and incubated at room temperature for $10 \mathrm{~min}$. After removing $20 \mu \mathrm{L}$ of extract, we stored the samples overnight at $-20^{\circ} \mathrm{C}$. After centrifugation at 4,000 rpm for $20 \mathrm{~min}$, the supernatants were transferred to 96 -well plates. We also prepared pooled quality control (QC) samples by combining equal aliquots of all extracts (Li et al., 2018; Gika et al., 2016). UPLC-QTOF/MS instrumentation and procedures

Chromatographic separations were performed on a UPLC system (SCIEX, UK) equipped with an ACQUITY UPLC T3 column $(100 \mathrm{~mm} \times 2.1 \mathrm{~mm}, 1.8 \mu \mathrm{m}$, Waters, UK). The column oven was maintained at a temperature of $35^{\circ} \mathrm{C}$. The mobile phase consisted of water (solvent A) and acetonitrile (solvent $\mathrm{B}$ ), each containing $0.1 \%$ formic acid ( $\mathrm{v} / \mathrm{v})$. We applied a $10 \mathrm{~min}$ gradient elution at a flow rate of $0.40 \mathrm{~mL} / \mathrm{min}$ as follows: $0 \sim 0.5 \mathrm{~min}, 5 \% \mathrm{~B} ; 0.5 \sim 7 \mathrm{~min}, 5 \%$ to $100 \% \mathrm{~B}$; 
119

120

121

122

123

124

125

126

127

128

129

130

131

132

133

134

135

136

137

138

139

140

141

142

143

144

145

146

147

148

149

150

151

152

153

154

155

156

157

158

7 8 $\mathrm{min}, 100 \% \mathrm{~B} ; 8 \sim 8.1 \mathrm{~min}, 100 \%$ to $5 \% \mathrm{~B}$; and $8.1 \sim 10 \mathrm{~min}, 5 \% \mathrm{~B}$. The injection volume for each sample was $4 \mu \mathrm{l}$. We used a triple TOF 5600 plus (SCIEX, UK) to detect metabolites eluted from the column. The Q-TOF was operated separately in both positive (ESI+) and negative (ESI-) electrospray ionization modes. The interface heater temperature was $650^{\circ} \mathrm{C}$ with a curtain gas pressure of $30 \mathrm{psi}$, and ion source gas 1 and ion source gas 2 were both set to 60 psi. The ion spray voltage was $5 \mathrm{kV}$ in the ESI+ mode and $4.5 \mathrm{kV}$ in the ESI- mode. The total cycle time was fixed at $0.56 \mathrm{~s}$. We summed four bins for each scan at a pulser frequency value of $11 \mathrm{kHz}$ by monitoring the $40 \mathrm{GHz}$ multichannel TDC detector with four-anode/channel detection. The dynamic exclusion was set at $4 \mathrm{~s}$. The mass spectrometric data were acquired in IDA mode, and the TOF mass range was between 60 and 1,200 Da. During the acquisition, we calibrated the mass accuracy every 20 samples. To further evaluate the stability of the liquid chromatographymass spectrometry (LC-MS) during the acquisition, we injected a QC sample after every 10 samples (Yu et al., 2018; Li et al., 2018).

\section{Data pre-processing}

We converted the LC-MS original wiff format files to mzXML files using ProteoWizard's msconvert tool (Adusumilli \& Parag, 2017), and processed them using XCMS, CAMERA, and metaX toolboxes in an R environment. The XCMS and CAMERA analysis matrix included the peak picking, peak grouping, retention time (RT) correction, and second peak grouping, as well as the isotope, adduct, and artifact annotations for each sample (Smith et al., 2006; Kuhl et al., 2012). The main XCMS parameter settings are provided in Table $S 2$. We identified each ion by combining the retention time and $\mathrm{m} / \mathrm{z}$ data. The intensity data from each peak were preprocessed using metaX to improve the quantitative information. We removed features that were detected in $<50 \%$ of QC samples or $80 \%$ of the biological samples, and extrapolated values for missing peaks using the k-nearest neighbor algorithm $(\mathrm{KNN})$ to further improve data quality. The QCbased robust LOESS signal correction was fitted to the QC data respecting the order of injection to minimize signal intensity drift over time. The relative standard deviations of the metabolic features were calculated across all QC samples, and we removed those were above 30\% (Xiao et al., 2012). The obtained features ( $\mathrm{m} / \mathrm{z}$ at a certain retention time) were queried against the Kyoto Encyclopedia of Genes and Genomes (KEGG, http://www.genome.jp/kegg/) and the Human Metabolome Database (HMDB, http:/www.hmdb.ca) (Kanehisa \& Goto, 2000). The mass errors for the database searches and fragment assignments were always below $10 \mathrm{ppm}$, indicating the reliability of elemental composition allocation. We used MS-DIAL to compare the MS/MS fragments of the metabolites against candidate molecules found in the HMDB, Massbank, and an internal fragment spectrum library (Tsugawa et al. 2015; Neumann \& Böcker, 2010).

\section{Statistical analysis}

We applied pareto scaling to the filtered data set, then conducted PCA and HCA using SIMCA-P Version 14.0 (Umetrics, Umeå, Sweden). A default sevenfold leave-one-out cross-validation method was employed to find the optimal model dimensionality and test the significance of the PCA models. We performed HCA clustering to establish the chemotypes of the core collections using the Euclidean distance matrix and the Ward linkage method (Saccenti et al., 2014). We

Peer) reviewing PDF | (2020:02:45831:2:0:NEW 15 Jul 2020) 
159

160

161

162

163

164

165

166

167

168

169

170

171

172

173

174

175

176

177

178

179

180

181

182

183

184

185

186

187

188

189

190

191

192

193

194

195

196

197

198

used SIMCA-P to construct the OPLS-DA model that could evaluate the differences in the established chemotypes. The model was first evaluated using $\mathrm{R}^{2} \mathrm{Y}$ (cum) and $\mathrm{Q}^{2} \mathrm{Y}$ (cum) values, and then using a permutation test to determine over-fitting. We determined latent biomarkers for each established chemotype using three criteria: (1) a variable importance of projection (VIP) value $>1$, (2) a Student's t-test $p$ value $<0.05$, and (3) a fold change (FC) value $>1.5$. These were displayed with large, dark-colored marks in volcano plots generated using SIMCA-P (Triba et al., 2015; Ding et al., 2018).

\section{Results}

\section{Metabolite annotation}

The total ion chromatograms (TICs) acquired in ESI+ and ESI- modes are shown in Figs.S2 and S3. We obtained 11,306 features (including 9,050 high-quality features) and 8,616 features (including 7,487 high-quality features) from the extracted ion chromatograms acquired in ESI+ and ESI- modes, respectively. Since a single isomer feature may match several metabolites from the first-order mass spectra, we calculated the mass feature number corresponding to the candidate metabolites. From the first-order mass spectra, we annotated 6,092 metabolites observed in ESI+ mode, and 3,554 metabolites observed in ESI- mode. From the secondary mass spectra, we annotated 451 and 325 metabolites in ESI+ and ESI- modes, respectively (Table 1). After KEGG pathway enrichment analysis, we found that the metabolites acquired in ESI+ and ESI- modes were involved in 69 and 78 metabolic pathways, respectively. The number of metabolites involved in the top 20 KEGG pathways is displayed in Fig. 1.

\section{Chemotype classification}

Since the metabolites annotated from the MS2 spectra were more accurate and unique than those annotated from the MS1 spectra, we used the MS2 annotation data for chemotype classification of the E. ulmoides samples. We followed two matrices: one using 132 samples $\times 451$ metabolites for the ESI+ mode data, and the other using 132 samples $\times 325$ metabolites for the ESI- mode data. The PCA score scatter plot and HCA dendrogram of the ESI+ mode data matrix can be found in Fig. 2. We used HCA to divide the 22 collections into four groups according to their chemotypes. The seven collections AG-1, BJ-5, MC, SNJ-2, SQ-1, SQ-2, and XS comprised chemotype I; the three collections BJ-3, CL, and HZ comprised chemotype II; the six collections BJ-1, BJ-2, BZ, LC, LY, and SNJ-1 comprised chemotype III; and the six collections AG-2, BJ4, JA, ZY-1, ZY-2, and ZZ comprised chemotype IV. The total variance of the data using the PCA model was $81.55 \%$, with $26.06 \%$ accounted for by principal component 1 (PC1) and $15.82 \%$ by principal component 2 (PC2). The two-dimensional PCA score plot provided a visualization of the separation and similarity across the chemical groups. Chemotype I was mainly placed on the left side along PC1 in the PCA score plot, chemotype IV was placed in the center, and chemotype III was placed on the right side. Meanwhile, along PC2, chemotype II was located in the upper half and the other three chemotypes were located in the lower half. Most biological duplications from each collection clustered together, and the boundaries of the classified chemotypes were evident in the PCA score plot, suggesting that the analytical procedures were consistent and repeatable. We did not observe separation based on the geographical origin of the 
199

200

201

202

203

204

205

206

207

208

209

210

211

212

213

214

215

216

217

218

219

220

221

222

223

224

225

226

227

228

229

230

231

232

233

234

235

236

237

238

germplasm collections from the analytical results, only that the collections from Shangqiu (SQ-1 and SQ-2) and Zunyi (ZY-1 and ZY-2) clustered in one chemotype.

We applied the PCA model to the data matrix of the ESI- mode data which accounted for $90.40 \%$ of the total variance, of which PC1 accounted for $24.36 \%$ and PC2 accounted for $16.34 \%$ (Fig. 3). Similar to the analysis of the data acquired in ESI+ mode, HCA divided the 22 collections into four chemotypes. Chemotype I was comprised of the five collections AG-1, AG2, JA, SNJ-2, and ZZ; chemotype II was comprised of the five collections BJ-2, BJ-4, LC, LY, and SNJ-1; chemotype III was comprised of the five collections BJ-5, MC, SQ-1, SQ-2, and XS; and chemotype IV was comprised of the seven collections BJ-1, BJ-3, B2, CL, HZ, ZY-1, and ZY-2. The classification results were slightly inconsistent with those from the ESI+ mode data, especially for chemotype I and chemotype IV. The ESI+ mode identified a higher number of metabolites than ESI- mode, and most metabolites including amino acids, short-chain carboxylic acids and flavonoids were detected in both modes. The chemotype classification boundaries at multiple dimensions in the PCA plots were unclear from the ESI- mode data (Figs.S4 and S5), so we ultimately selected and adopted the classification results originating from the ESI+ mode to establish the chemotype for the collections.

To determine whether a certain classified chemotype of the male Eucommia core collections was unique enough to be distinguished from other types, we constructed four supervised OPLSDA models to provide further insights into the separation (Fig. 4). To assess each OPLS-DA model, we calculated $\mathrm{R}^{2} \mathrm{Y}$ (cum) and $\mathrm{Q}^{2} \mathrm{Y}$ (cum) coupled with two indicators referring to the intercept of $\mathrm{R}^{2}$ and $\mathrm{Q}^{2}$ from the permutation test (Table 2). We used the generally accepted threshold values $(>0.5)$ of $\mathrm{R}^{2} \mathrm{Y}$ (cum) and $\mathrm{Q}^{2} \mathrm{Y}$ (cum) to judge whether the OPLS-DA model was stable and effective (Triba et al., 2015), and clearly found that the constructed models achieved these standards, confirming that each classified chemotype had a distinct metabolite composition. However, the permutation test showed that the OPLS-DA model between chemotype IV and the other chemotypes had slightly higher $\mathrm{R}^{2}$ and $\mathrm{Q}^{2}$ intercept values, suggesting the possibility of over-fitting in that model.

\section{Screening chemical markers}

We determined the most relevant chemical markers for discriminating each chemotype based on the VIP value, p-value, and FC value in the OPLS-DA model. In total, we screened 29 chemical markers, 21 of which corresponded to chemotype I, six corresponded to chemotype II, 19 corresponded to chemotype III, and five corresponded to chemotype IV (Fig. 5 and Table S3). The chemical markers were flavonoids, amino acids and their derivatives, cinnamic acids and their derivatives, lipids, alkaloids, organooxygen compounds, and organoheterocyclic compounds. The overlapping correlation of the markers is illustrated in Fig. 6. For chemotype I, the exclusive markers were cinnamic acid, L-phenylalanine, glycyl -L-leucine, and jasmonic acid. For chemotype III, the exclusive markers were S-adenosyl-L-methionine and astragalin. Chemotype II and chemotype IV each contained only one exclusive chemical marker, carnosic acid and isorhamnetin, respectively. The chemical marker variations and the peak intensities in the four chemotypes are shown as box-and-whisker plots (Fig. S6). To re-examine the validity of 
239 the markers in denoting different chemotypes, we built a data matrix consisting of the 29 markers

240 and 22 collections. We used the same clustering method to draw a heat map (Fig. 7) that showed

241 the samples were well-classified and in agreement with the chemotypes established above.

242 However, the HZ collections were misclassified.

\section{Discussion}

244 E. ulmoides, a monotypic genus of Eucommiaceae, has long been considered to have relatively

245 limited intraspecific variations compared to other species belonging to multi-type genera. In the

2461980 s, there was high demand for E. ulmoides bark, significantly increasing its market value.

247 Germplasm resources, especially aged E. ulmoides trees, were nearly devastated, leading many

248 researchers to believe that the species' wild resources had been exhausted in China (Deng et al.,

249 2006; Wang et al., 2006). The majority of existing trees were planted from homogeneous

250 provenances and propagated by grafting, which was previously believed to limit genetic diversity

251 (Wang et al, 2006). The sustainable cultivation and utilization of the present resources and

252 effective breeding is one of the major concerns of E. ulmoides researchers. The first steps should

253 be the implementation and evaluation of the species variations at phenotypic, genotypic, and

254 chemotypic levels. A previous study conducted high-resolution metabolic phenotyping using

255 metabolomic approaches to assess a broad range of possible metabolites and enable informed

256 selection in plants (Fernie \& Schauer, 2009). Our work was the first attempt at disclosing the

257 metabolome of male E. ulmoides flowers and the chemical variations of the core collections from

258 different geographical origins under similar cultivation measures. These results could provide a

259 foundation for further research on the medical use of these male flowers and their metabolic

260 network and regulation.

261 Due to LC-MS instruments' versatility and sensitivity, they can detect hundreds to thousands

262 of features from a single sample, and, collectively, tens of millions of features for large-scale

263 projects in non-targeted metabolomics studies (Rocca-Serra et al., 2016). A significant portion

264 of the detected metabolomes were highly redundant due to the presence of isotopes, adducts, and

265 in-source fragments (Kachman et al., 2019). Robust annotation and identification of metabolites

266 in non-targeted studies remains a major challenge in LC-MS-based non-targeted metabolomics,

267 which might lead to false discoveries and incorrect interpretations (Salek et al., 2013; Scheubert

268 et al., 2017). Other issues, including matching multiple identities with a single feature and

269 multiple disagreeing annotations, often arise when matching the detected features to candidate

270 chemical structures based on their mass and predicting fragmentation patterns based on chemical

271 libraries (Alden et al, 2017). However, it is not realistic for most individual laboratories to

272 establish a comprehensive spectral library that encompasses all possible chemical standards

273 (Broeckling et alo, 2016). Although most modern validation strategies for non-targeted

274 approaches are not well defined ( $\mathrm{Naz}$ et al., 2014), the metabolites annotated in our study cover

275 most well-known chemical constituent categories, provide the metabolomic profiles for male $E$.

276 ulmoides flowers, and present a potential holistic approach to improving germplasm resource

277 evaluation and understanding its physiological mechanisms. 
278

279

280

281

282

283

284

285

286

287

288

289

290

291

292

293

294

295

296

297

298

299

300

301

302

303

304

305

306

307

308

309

310

311

312

313

314

315

316

317

The unsupervised clustering HCA method coupled with PCA reduced the dimensionality of the multivariate data and created new principal components using linear combinations to determine similarities and differences across the samples. These methods have been applied in previous plant metabolomic analyses (Gad et al., 2013; Cevallos-Cevallos et al., 2009). Furthermore, the combined application of PCA and OPLS-DA to spectral datasets yielded valuable insights into general spectral trends and group predictive spectral features (Worley \& Powers, 2016). The core collections are small sets of accessions chosen to represent the genetic spectrum of the whole collection and cover high variation levels, representing up to $70 \%$ of the genetic diversity of a species. We used multivariate methods to objectively classify the four chemotypes of the male $E$. ulmoides core collections, referencing the main chemical variation forms in the germplasm resource. It should be noted that the biological replicates from the six collections SNJ-1, BZ, BJ-3, BJ-1, HZ, and LY were not clustered within one chemotype. In addition to errors resulting from using the employed clustering method, we assumed that some random sampling errors, injection problems, and other instrument noises or drifts were the main factors affecting the clear classification of the six collections' biological replicates. However, according to our Spearman's correlation analysis based on the peak intensity data, the correlation coefficient was around or above $90 \%$, and we observed a higher correlation in replicates of the same collection than in different collections (Fig. S7). This indicated that there were significant similarities in the collection duplications and they could therefore be identified as belonging to the same chemotype. Due to their different physicochemical properties (e.g., polarity), the two data sets detected using the ESI+ and ESI- modes were also different to some extent, leading to slightly inconsistent HCA chemotype classification. Because of the great dimension and complexity of a non-targeted metabolomics data matrix, it is hard to obtain a strictly precise classification using existing bioinformatics tools. In this study, we verified the effectiveness of the classification results using the OPLA-DA model. However, both classified chemotype sets were inconsistent with geographical origin patterns. We inferred that the chemical variation may have originated from the inherent genetic variation of the germplasm and intrapopulation (not interpopulation) variation. Similar results at the genetic level were found in previous studies (Wang et al., 2006; Wu, 2014).

Other studies have explored the main categories of the nutritive components of male $E$. ulmoides flowers, including iridoids, phenylpropanoids, flavonoids, amino acid derivatives, and phenolic glycosides (Yan et al., 2018; Ding et al., 2014). In our current study, we screened out 29 chemical biomarkers belonging to seven metabolite classes, the majority of which were flavonoids and amino acid derivatives. Comparison and variation analyses on the 17 amino acids in E. ulmoides male flowers of 193 germplasm resources showed that the content variation degrees, evaluated by coefficient of variation (CV) values, were relatively higher in proline (Pro), cysteine (Cys), lysine (Lys), and methionine (Met) (Du et al., 2016). Another study evaluated the content variations of the eight major active components in the same resources, and found that geniposide, geniposidic acid, and astragaline had the highest variation degrees (CV values above $80 \%$ ); followed by isoquercitrin, chlorogenic acid, and aucubin (CV values around 50\%); and

Peer] reviewing PDF | (2020:02:45831:2:0:NEW 15 Jul 2020) 
318 total flavonoids and total amino acids had the lowest variation degrees ( $\mathrm{CV}$ values below 25\%) 319 (Du et al., 2017). We assumed that the chemical components with the highest degrees of content 320 variation in the germplasm resources would be potential biomarkers for chemotype differences, 321 and biomarkers such as chlorogenic acid, methionine, and S-adenosyl-L-methionine complied 322 with this assumption. However, the most likely biomarkers geniposide, geniposidic acid, and

323

324

325

326

327

328

329

330

331

332

333

334

335

336

337

338

339

340

341

342

343

344

345

346

347

348

349

350

351

352

353

354

355

356

357

358 astragaline did not comply with our results. One possible reason for these inconsistencies may be that the potential biomarkers' features were not fully annotated because the coverage of standard spectra in the reference database was incomplete. Additionally, these were two studies conducted using different experimental conditions, compound extraction procedures, and measuring instruments. Future studies should validate the identification and structure of the screened biomarkers using multiple optimized in silico tools, and confirm their results using classical targeted analytical approaches such as the NMR technique to get more reliable and reproducible results.

\section{Conclusion}

We determined the metabolite profiles of male E. ulmoides flowers from different geographical origins using a developed UPLC-QTOF/MS fingerprinting method. We found abundant compositional variations and performed phenotyping at the metabolic level across several male $E$. ulmoides core collections, providing a basis for understanding the formation mechanism of economically important traits. A total of 451 and 325 metabolites were identified in ESI+ and ESI- modes, respectively, from 22 male flower core collections. We coupled the multivariate statistical method PCA with OPLS-DA models to provide a new technique for classifying $E$. ulmoides germplasm resources that is efficient in revealing the major metabolites that contribute to chemotype discrimination. Four chemotypes could be determined from the MS2-annotated metabolites that were detected in ESI+ mode. We proposed that compositional variations across the chemotypes were due to genetic composition variations instead of geographical distributions. We screened a total of 29 robust chemical markers that enabled the differentiation of four established chemotypes, most of which were flavonoids and amino acid derivatives. The newly proposed chemical markers, along with existing monograph and molecular markers, could be utilized for more precise germplasm resource identification. It should be noted that our results are preliminary and require further verification using a larger set of samples.

\section{References}

Adusumilli R, Parag M. 2017. Proteomics. Humana Press: New York.

Afzan A, Kasim N, Ismail NH, Azmi N, Ali AM, Mat N, Wolfender, J. 2019. Differentiation of Ficus deltoidea varieties and chemical marker determination by UHPLC-TOFMS metabolomics for establishing quality control criteria of this popular Malaysian medicinal herb. Metabolomics 15(35):1-11 DOI 10.1007/s11306-019-1489-2.

Alden N, Krishnan S, Porokhin V, Raju R, McElearney K, Gilbert A, Lee K. 2017. Biologically consistent annotation of metabolomics data. Analytical Chemistry 89:1309713104 DOI 10.1021/acs.analchem.7b02162.

Broeckling CD, Ganna A, Layer M, Brown K, Sutton B, Ingelsson E, Peers G, Prenni JE. 2016. Enabling efficient and confident annotation of LC-MS metabolomics data through 
MS1 spectrum and time prediction. Analytical Chemistry 88:9226-9234 DOI 10.1021/acs.analchem.6b02479.

Call VB, Dilcher DL. 1997. The fossil record of Eucommia (Eucommiaceae) in North America. American Journal of Botany 84:798-814.

Cevallos-Cevallos JM, Reyes-De-Corcuera JI, Etxeberria E, Danyluk MD, Rodrick GE. 2009. Metabolomic analysis in food science: a review. Trends in Food Science \& Technology 20:557-566 DOI 10.1016/j.tifs.2009.07.002.

Da Silva GS, Canuto KM, Ribeiro PRV, de Brito ES, Nascimento MM, Zocolo GJ, Coutinho JP, de Jesus RM. 2017. Chemical profiling of guarana seeds ( Paullinia cupana) from different geographical origins using UPLC-QTOF-MS combined with chemometrics. Food Research International 102:700-709 DOI 10.1016/j.foodres.2017.09.055.

Deng JY, Li JQ, Huang HW. 2006. Discovery of an originally wild tree of Eucommia ulmoides Oliver by AFLP fingerprinting. Journal of Wuhan Botanical Research 24:509-513[in Chinese].

Ding MY, Jiang Y, Yu X, Zhang D, Li J, Wang H, Shen JY, Gao XM, Chang, YX. 2018. Screening of combinatorial quality markers for natural products by metabolomics coupled with chemometrics. A case study on Pollen Typhae. Frontiers in Pharmacology 9:691 DOI 10.3389/fphar.2018.00691.

Ding YX, Guo YJ, Ren YL, Dou DQ, Li Q. 2014. Isolation of flavonoids from male flowers of Eucommia ulmoides and their anti-oxidantive activities. Chinese Traditional and Herbal Drugs 45:323-327[in Chinese].

Dong JE, Liang ZS, Zhang KJ, Sun, SH. 2005. Dynamic changes of secondary metabolites in Eucommia ulmoides male flower. Joural of Plant Resources and Enviroment 4:7-10[in Chinese].

Du HY, Hu WZ, Yu R. 2013. The report on development of China's Eucommia rubber resources and industry (2013). Social Science Academic Press: Beijing [in Chinese].

Du HY, Hu WZ, Yu R. 2015. The report on development of China's Eucommia rubber resources and industry (2014-2015). Social Science Academic Press: Beijing [in Chinese].

Du QX, Liu PF, Wei YX, Qing J, Du HY. 2016. Comprehensive evaluation of Eucommia ulmoides male flowers quality by principal component and cluster analysis. Bulletin of Botanical Research 36:846-852[in Chinese].

Du QX, Wei YX, Liu PF, Qing, J, Du HY. 2017. Principal component analysis and overall evaluation of amino acids in Eucommia ulmoides male flowers. Journal of south China agricultural university 38:76-81[in Chinese].

Du QX, Wei YX, Liu PF, Du HY. 2017. Diversity of the content of main active components in Eucommia ulmoides male flowers. Scientia silvae sinicae 53:35-43[in Chinese].

Fan SC, Gao Y, Zhang HZ, Huang M, Bi HC. 2017. Untargeted and targeted metabolomics and their applications in discovering drug targets. Progress in Pharmaceutical Sciences 41:263-269[in Chinese].

Feng F, Liang ZR. 1996. Understanding and utilization of Eucommia ulmoides in Chinese history. Journal of Northwest Forestry College 2:84-89[in Chinese].

Fernie AR, Schauer N. 2009. Metabolomics-assisted breeding: a viable option for crop improvement? Trends in Genetics 25:39-48 DOI 10.1016/j.tig.2008.10.010.

Gad HA, El Ahmady SH, Abou Shoer MI, Al Azizi, MM. 2013. Application of chemometrics in authentication of herbal medicines: a review. Phytochemical Analysis 24:1-24.

Gika HG, Zisi C, Theodoridis G, Wilson ID. 2016. Protocol for quality control in metabolic 
405

406

407

408

409

410

411

412

413

414

415

416

417

418

419

420

421

422

423

424

425

426

427

428

429

430

431

432

433

434

435

436

437

438

439

440

441

442

443

444

445

446

447

448

449

450

profiling of biological fluids by U (H) PLC-MS. Journal of Chromatography B 1008:15-25 DOI 10.1016/j.jchromb.2015.10.045.

Kachman M, Habra H, Duren W, Wigginton J, Sajjakulnukit P, Michailidis G, Burant C, Karnovsky A. 2019. Deep annotation of untargeted LC-MS metabolomics data with Binner. Bioinformatics. DOI 10.1093/bioinformatics/btz798.

Kanehisa M, Goto S. 2000. KEGG: kyoto encyclopedia of genes and genomes. Nucleic Acids Research 28(1):27-30 DOI 10.1093/nar/28.1.27.

Kuhl C, Tautenhahn R, Bottcher C, Larson TR, Neumann S. 2012. CAMERA: an integrated strategy for compound spectra extraction and annotation of liquid chromatography/mass spectrometry data sets. Analytical chemistry 84(1):283-289 DOI 10.1021/ac202450g.

Li HG, Du HY, Jia HY, Chen HH, Xu JH, Du QX, Wang, L. 2018. Establishment of male core collection of Eucommia ulmoides based on phenotypic traits. Molecular Plant Breeding 16:591-601[in Chinese].

Li HG, Xu JH, Du HY, Wuyun TN, Liu PF, Du, QX. 2018. Preliminary construction of core collection of Eucommia ulmoides based on allele number maximization strategy. Scientia Silvae Sinicae 54:42-51[in Chinese].

Li YK, Fang JH, Qi XJ, Lin MM, Zhong YP, Sun LM, Cui W. 2018. Combined analysis of the fruit metabolome and transcriptome reveals candidate genes involved in flavonoid biosynthesis in Actinidia Arguta. International Journal of Molecular Sciences 19:1471 DOI 10.3390/ijms19051471.

Liang JN. 2007. The study on the morphological development of flower and the ultrastructure of pollen development in Eucommia ulmoides Oliver. Beijing Normal University [in Chinese].

Liu CH. 2010. Floral organogenesis and phylogenetic analysis in Eucommia ulmoides Oilv. Peking University, Beijing [in Chinese].

National Health Commission. Announcement on the approval of six new food raw materials including chitooligosaccharide etc. Available at http://www.nhc.gov.cn/sps/s7890/201405/367ce408981e4807809e107417b3d361.shtml. (accessed 10/16 2019). [in Chinese].

Naz S, Vallejo M, García A, Barbas C. 2014. Method validation strategies involved in nontargeted metabolomics. Journal of Chromatography A 1353:99-105 DOI 10.1016/j.chroma.2014.04.071.

Neumann S, Böcker S. 2010. Computational mass spectrometry for metabolomics: identification of metabolites and small molecules. Analytical and Bioanalytical Chemistry 398:2779-2788 DOI 10.1007/s00216-010-4142-5.

Ning ZC, Lu C., Zhang YX, Zhao SY, Liu BQ, Xu XG, Liu YY. 2013. Application of plant metabonomics in quality assessment for large-scale production of traditional Chinese medicine. Planta Medica 79:897-908 DOI 10.1055/s-0032-1328656.

Rocca-Serra P, Salek RM, Arita M, Correa E, Dayalan S, Gonzalez-Beltran A, Ebbels T, Goodacre R, Hastings J, Haug K. 2016. Data standards can boost metabolomics research, and if there is a will, there is a way. Metabolomics 12:14 DOI 10.1007/s11306-015-0879-3.

Saccenti E, Hoefsloot HC, Smilde AK, Westerhuis JA, Hendriks MM. 2014. Reflections on univariate and multivariate analysis of metabolomics data. Metabolomics 10:361-374 DOI 10.1007/s11306-013-0598-6.

Salek RM, Steinbeck C, Viant MR, Goodacre R, Dunn WB. 2013. The role of reporting standards for metabolite annotation and identification in metabolomic studies. GigaScience 2:2047-2217. 
451

452

453

454

455

456

457

458

459

460

461

462

463

464

465

466

467

468

469

470

471

472

473

474

475

476

477

478

479

480

481

482

483

484

485

486

487

488

489

490

491

492

493

Scheubert K, Hufsky F, Petras D, Wang M, Nothias L, Dührkop K, Bandeira N, Dorrestein PC, Böcker S. 2017. Significance estimation for large scale metabolomics annotations by spectral matching. Nature Communications 8:1-10 DOI 10.1038/s41467-017-01318-5.

Smith CA, Want EJ, O'Maille G, Abagyan R, Siuzdak, G. 2006. XCMS: processing mass spectrometry data for metabolite profiling using nonlinear peak alignment, matching, and identification. Analytical Chemistry 78(3): 779-787 DOI 10.1021/ac051437y.

Taamalli A, Iswaldi I, Arráez-Román D, Segura-Carretero A, Fernández-Gutiérrez A, Zarrouk M. 2014. UPLC-QTOF/MS for a rapid characterisation of phenolic compounds from leaves of Myrtus Communis L. Phytochemical Analysis 25:89-96 DOI $10.1002 /$ pca.2475.

Triba MN, Le Moyec L, Amathieu R, Goossens C, Bouchemal N, Nahon P, Rutledge DN, Savarin P. 2015. PLS/OPLS models in metabolomics: the impact of permutation of dataset rows on the K-fold cross-validation quality parameters. Molecular BioSystems 11:13-19 DOI 10.1039/C4MB00414K.

Tsugawa H, Cajka T, Kind T, Ma Y, Higgins B, Ikeda K, Kanazawa M, VanderGheynst J, Fiehn O, Arita M. 2015. MS-DIAL: data-independent MS/MS deconvolution for comprehensive metabolome analysis. Nature methods 12(6):523 DOI 10.1038/nmeth.3393.

Wang AQ, Huang LQ, Shao AJ, Cui GH, Chen M, Tong CH. 2006. Genetic diversity of Eucommia ulmoides by RAPD analysis. China Journal of Chinese Materia Medica 31:1583-1586 [in Chinese].

Wolfender JL, Glauser G, Grata E, Rudaz S. 2008. UPLC-TOF-MS for high resolution plant metabolite profiling and metabolomics. Planta Medica 74:L54.

Worley B, Powers R. 2016. PCA as a practical indicator of OPLS-DA model reliability. Current Metabolomics 4:97-103 DOI 10.2174/2213235X04666160613122429.

Wu M. 2014. Development of genomic SSR markers and genetic diversity evaluation of Eucommia ulmoides Oliv. Chinese Academy of Forestry [in Chinese].

Xiao JF, Zhou B, Ressom HW. 2012. Metabolite identification and quantitation in LC-MS/MSbased metabolomics. TrAC Trends in Analytical Chemistry 32:1-14. DOI 10.1016/j.trac.2011.08.009.

Yan Y, Zhao H, Zou LS, Liu XH, Chai C, Wang SN, Hua YJ. 2018. Analysis of chemical constituents in Eucommiae flos male by LC- ESI-Triple TOF MS/MS. Food Science:215221 [in Chinese].

Yu CN, Luo XJ, Zhan XR, Hao J, Zhang L, L Song YB, Shen CJ, Dong M. 2018. Comparative metabolomics reveals the metabolic variations between two endangered Taxus species (T. fuana and T. yunnanensis) in the Himalayas. Bmc Plant Biology 18:197 DOI 10.1186/s12870-018-1412-4.

Zhang Q, Su YQ, Yang FX, Peng JN, Li XH, Sun RC. 2007. Antioxidative activity of water extracts from leaf, male flower, raw cortex and fruit of Eucommia ulmoides Oliv. Forest Products Journal 57(12): 74+.

Zhu HL, Lin HQ, Tan J, Wang CZ, Wang H, Wu FL, Dong QH, Liu YH, Li PY, Liu, JP. 2019. Uplc-Qtof/Ms-based non targeted metabolomic analysis of mountain- and gardencultivated ginseng of different ages in northeast China. Molecules 24:33. DOI $10.3390 /$ molecules 24010033 .

Peer) reviewing PDF | (2020:02:45831:2:0:NEW 15 Jul 2020) 


\section{Figure 1}

Number of annotated metabolites involved in the top 20 KEGG pathways by the MS2 spectra. (A) under the ESI+ mode; (B) under the ESI- mode.

(A) under the ESI+ mode; (B) under the ESI- mode.

A

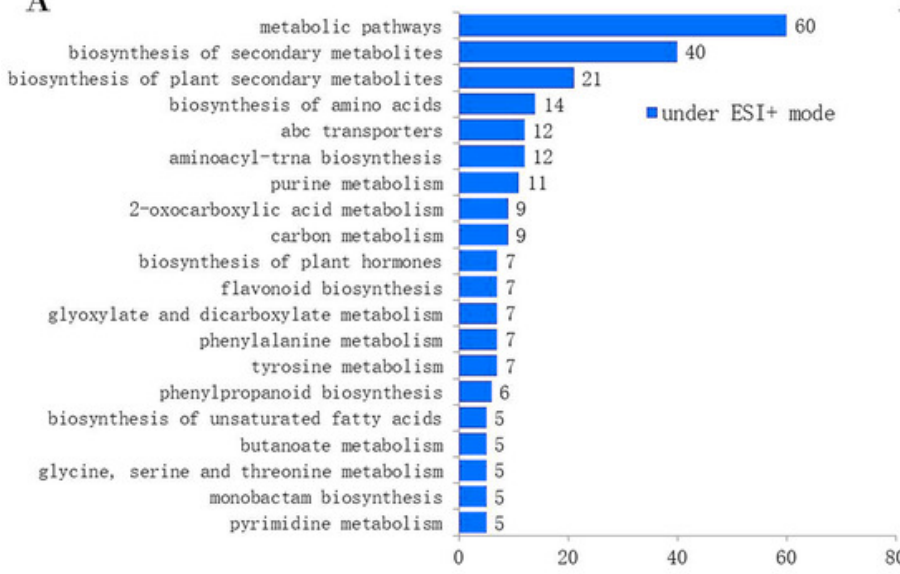

B

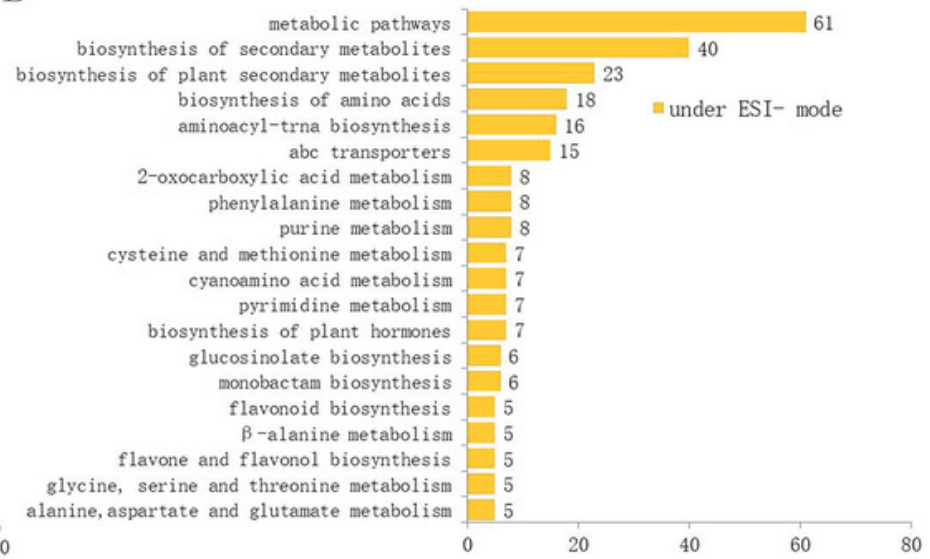


Figure 2

Chemotype classification of 22 male E. ulmoides core collections by PCA and HCA basing on metabolomics data acquired in the ESI+ mode.

(A) PCA score plot of 132 samples, with PC1 accounting for $26.06 \%$ of the total variance and PC2 explaining $15.82 \%$. The plot ellipse represents 95\% confidence region for Hotelling's T2;

(B) HCA dendrogram by using the Euclidean distance matrix and Ward's linkage method. 
A

AG-1

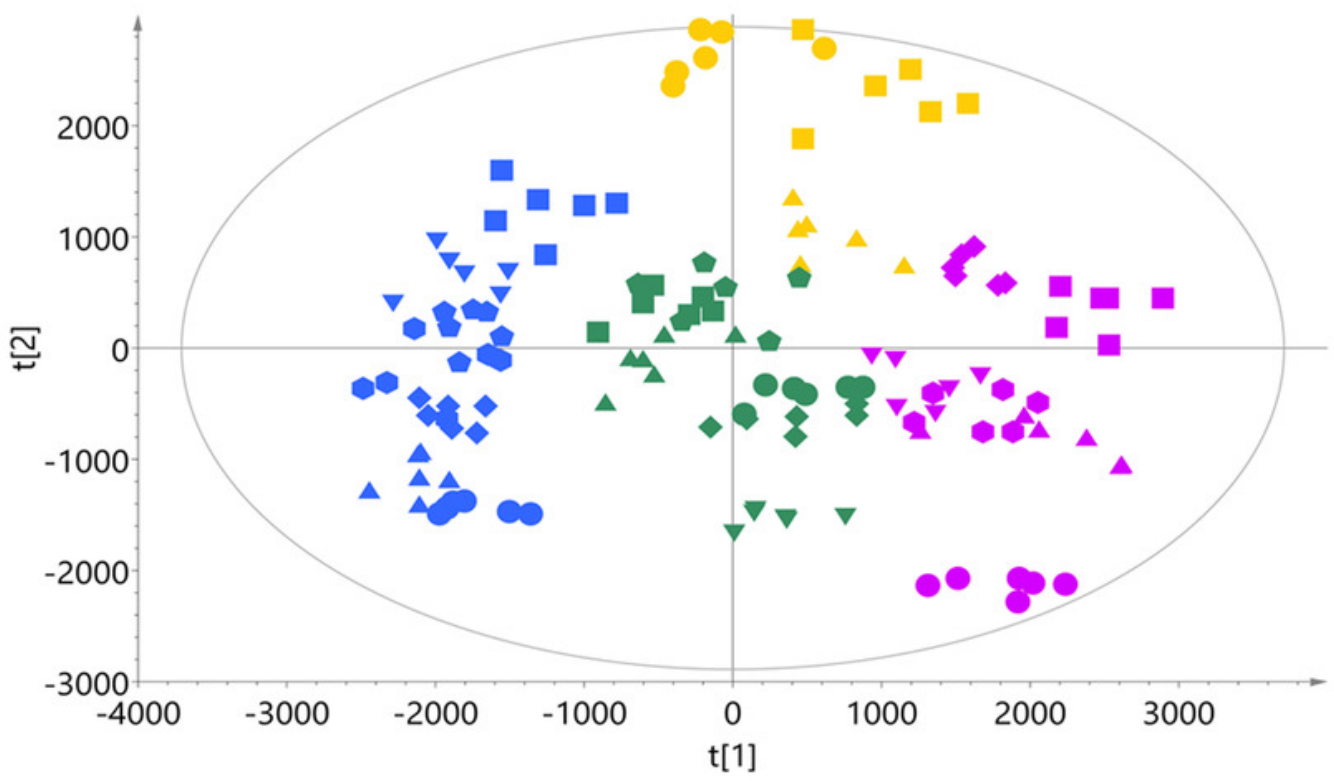

$\nabla B J-2$

$B J-3$

$\nabla \mathrm{BJ}-4$

BJ-5

$\square \mathrm{BZ}$

$\odot \mathrm{CL}$

$\triangle \mathrm{HZ}$

JA

OLC

$\Delta \mathrm{LY}$

$\nabla \mathrm{MC}$

SNJ-1

$\triangle \mathrm{SNJ}-2$

$\square \mathrm{SQ}-1$

SQ-2

$\rightarrow \mathrm{XS}$

$\Delta \mathrm{ZY}-1$

$\mathrm{ZY}-2$

$\mathrm{ZZ}$

B

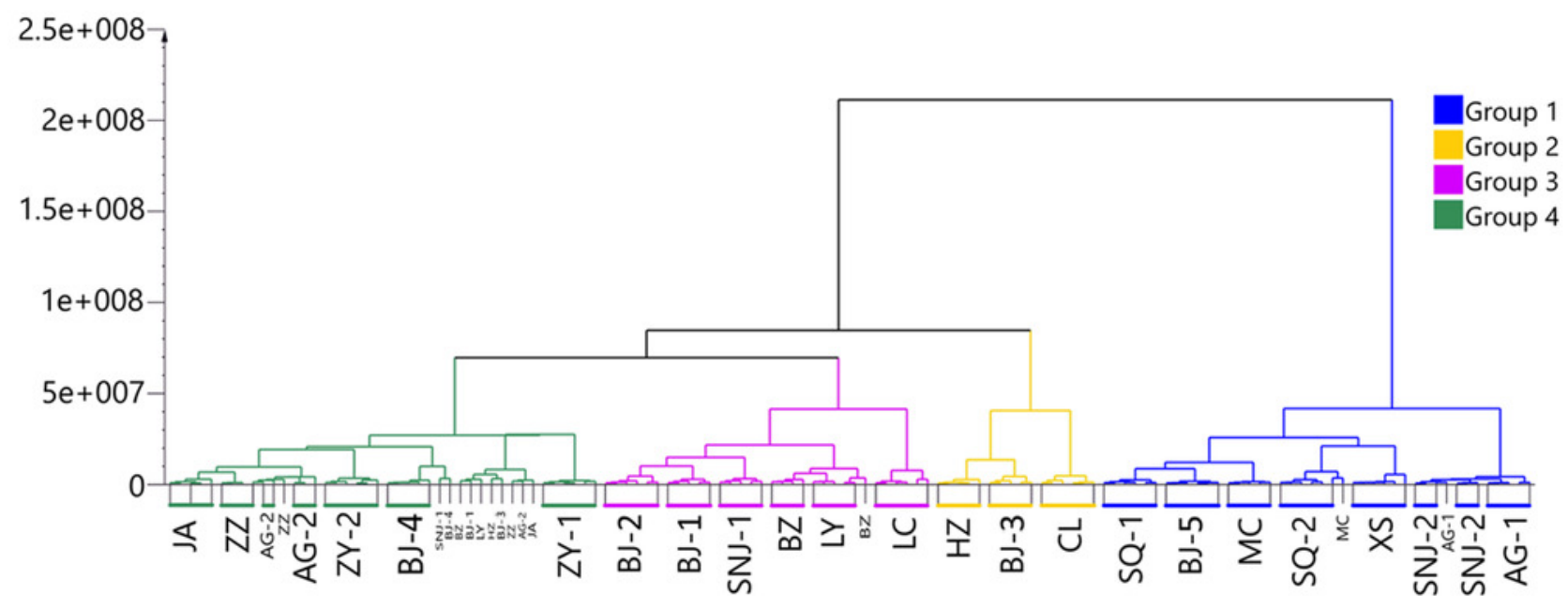




\section{Figure 3}

Chemotype classification of 22 male E. ulmoides core collections by PCA and HCA basing on metabolomics data acquired in ESI- mode.

(A) PCA score plot of 132 samples, with PC1 accounting for $24.36 \%$ of the total variance and PC2 explaining $16.34 \%$. The plot ellipse represents the $95 \%$ confidence region for Hotelling's T2; (B) HCA dendrogram by using the Euclidean distance matrix and Ward's linkage method. 
A

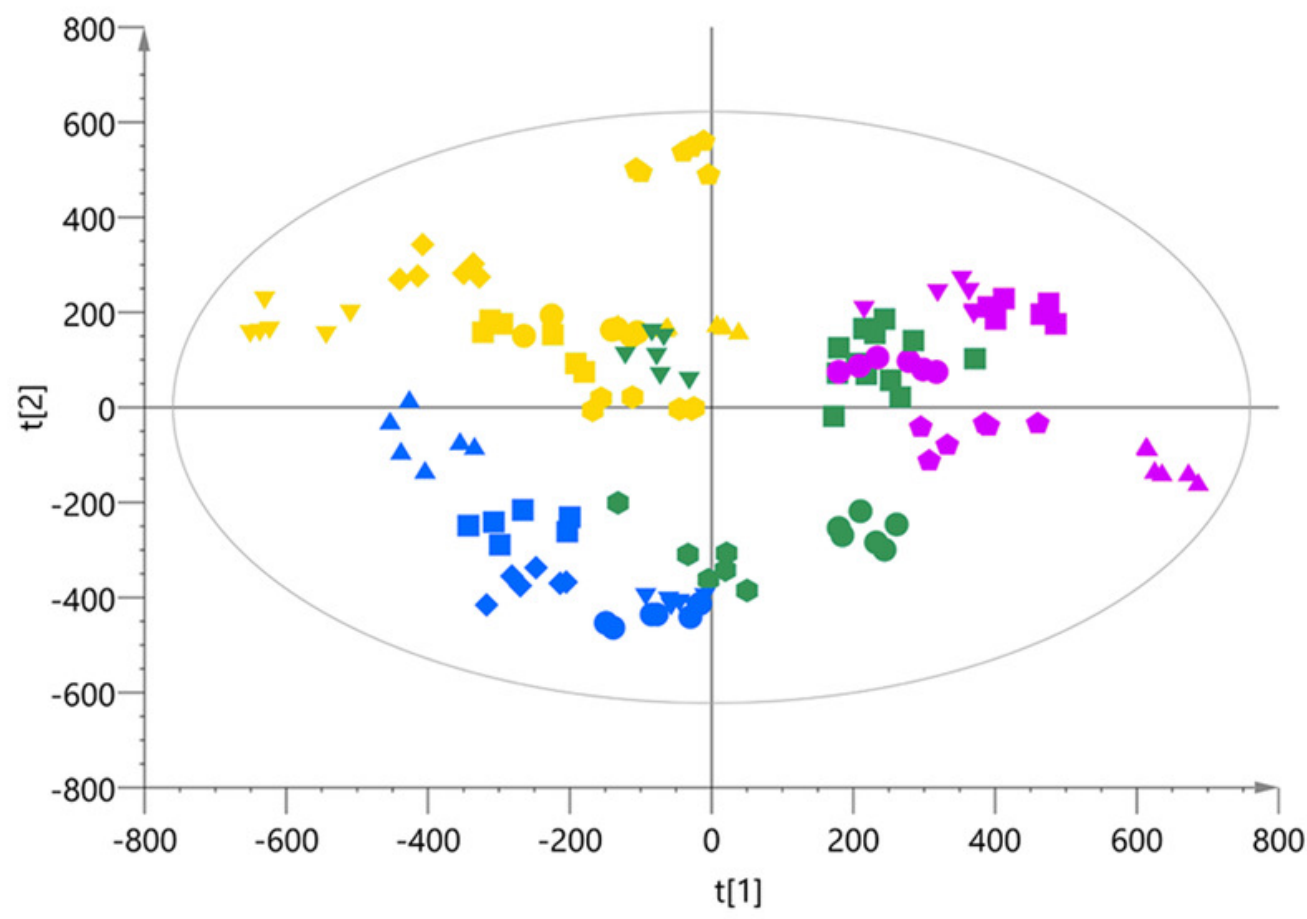

AG-1

AG-2

$\triangle \mathrm{BJ}-1$

$\mathrm{BJ}-2$

BJ-3

BJ -4

BJ -5

$\mathrm{BZ}$

$\nabla \mathrm{CL}$

$\mathrm{HZ}$

IA

$\Delta \mathrm{LC}$

$\nabla \mathrm{LY}$

$\triangle \mathrm{MC}$

SNJ-1

SNJ-2

$\triangle \mathrm{SQ}-1$

$\nabla \mathrm{SQ}-2$

XS

$\mathrm{ZY}-1$

$\mathrm{ZY}-2$

$\nabla \mathrm{ZZ}$

B

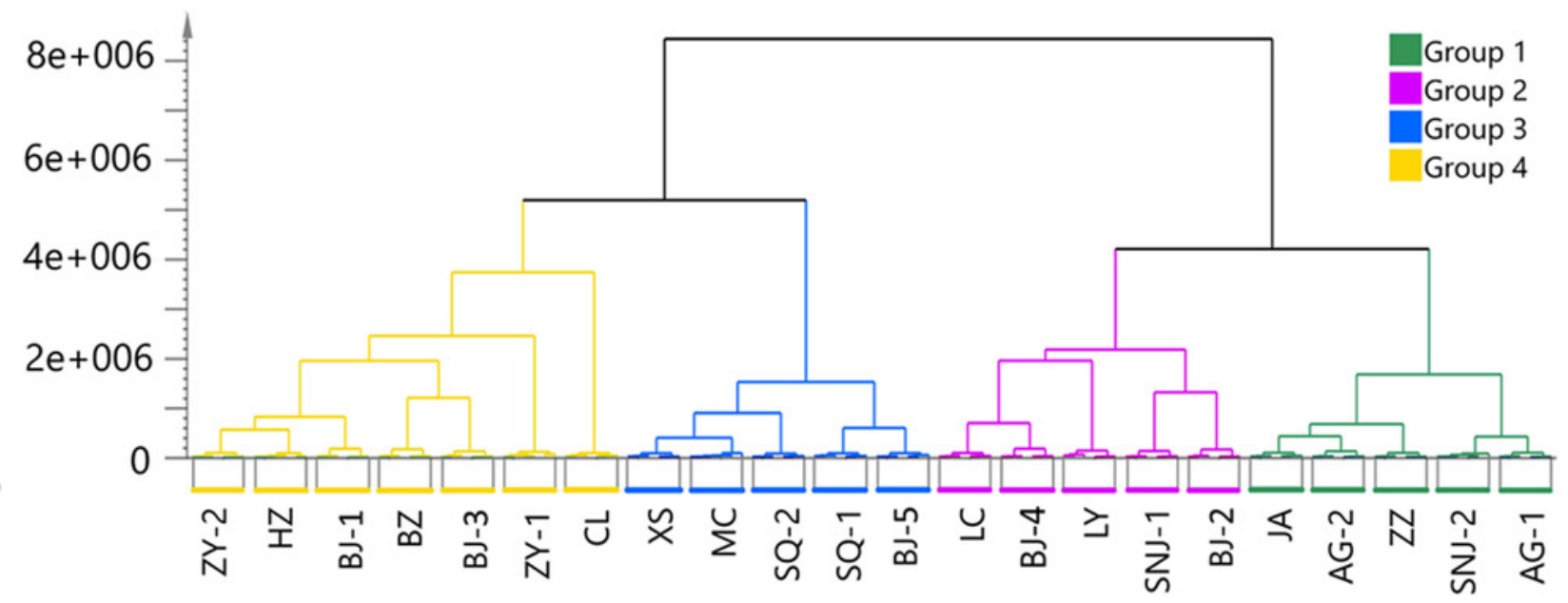


Figure 4

OPLS-DA score plots and permutation test plots of the classified chemotype against the other types.

$(A) \&(B)$ chemotype I against the other types; $(C) \&(D)$ chemotype II against the other types; $(E) \&(F)$ chemotype III against the other types; $(G) \&(H)$ chemotype IV against the other types. 
A $\square$ chemotype I the other chemotypes

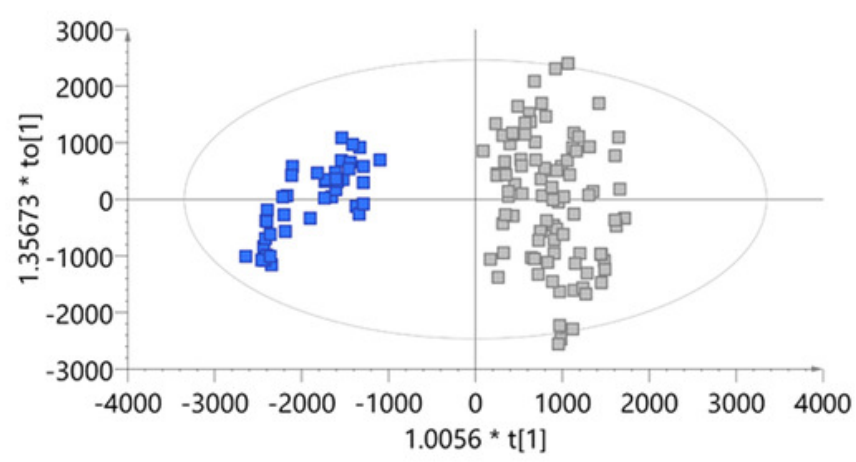

C the other chemotypes chemotype II

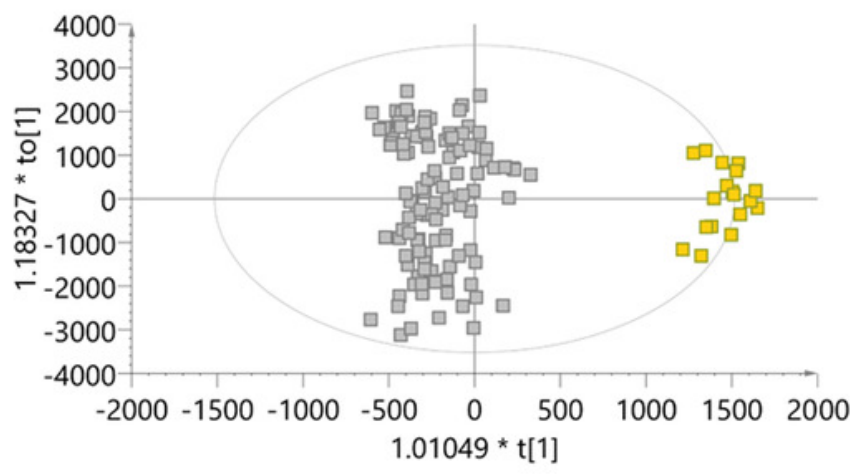

E chemotype III the other chemotypes

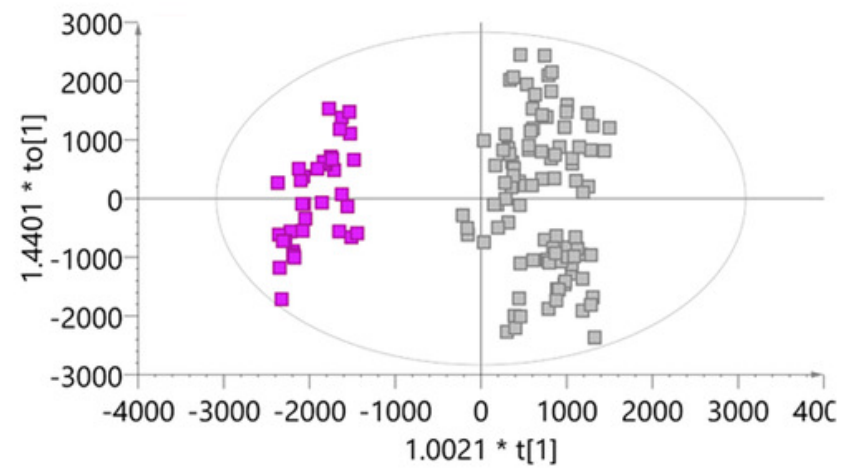

G the other chemotypes $\square$ chemotype IV

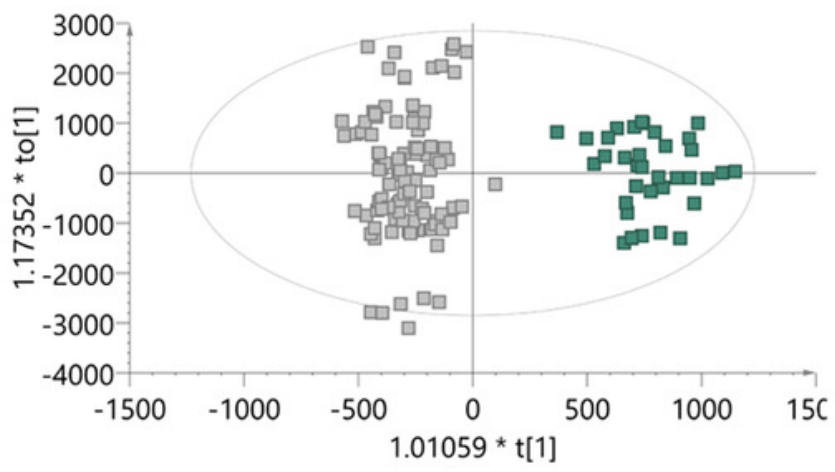

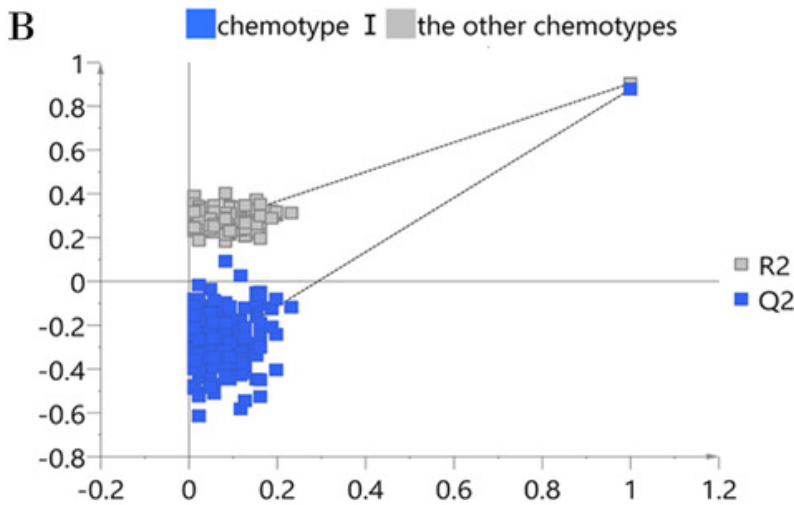

D the other chemotypes chemotype $\mathbb{I}$

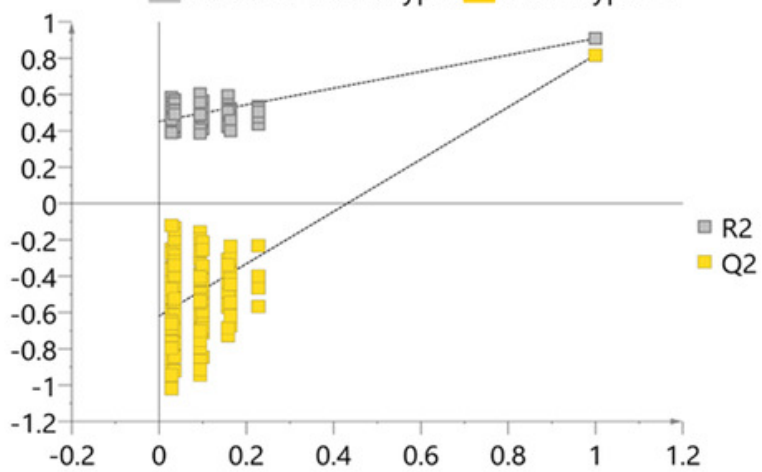

F $\quad$ chemotype III the other chemotypes

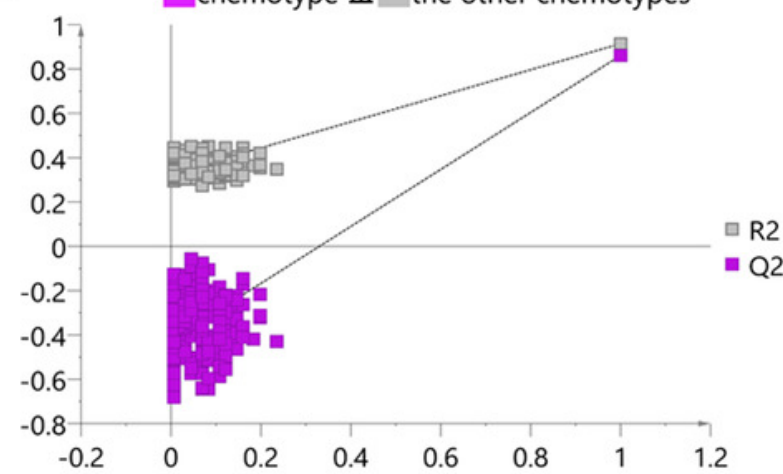

H the other chemotypes chemotype IV

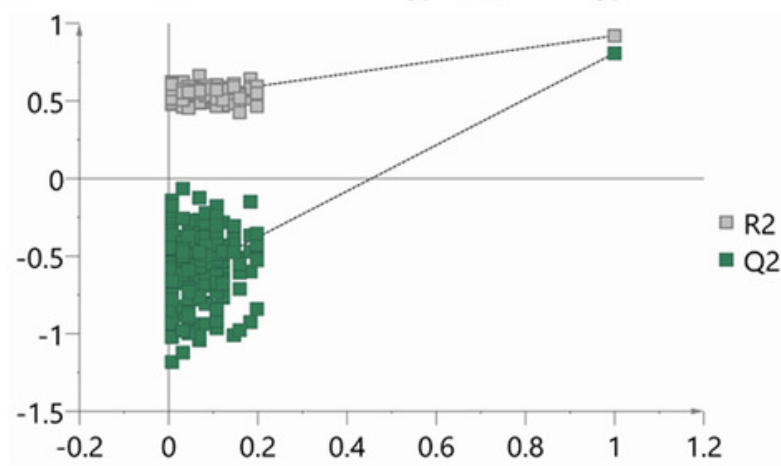


Figure 5

Volcano plots of chemical markers screened for each classified chemotype.

The $\mathrm{X}$-axis corresponds to $\log 2(\mathrm{FC}$ value), and the $\mathrm{Y}$-axis corresponds to $-\lg (\mathrm{p}$ value). (A) chemotype I; (B) chemotype II; (C) chemotype III; (D) chemotype IV.
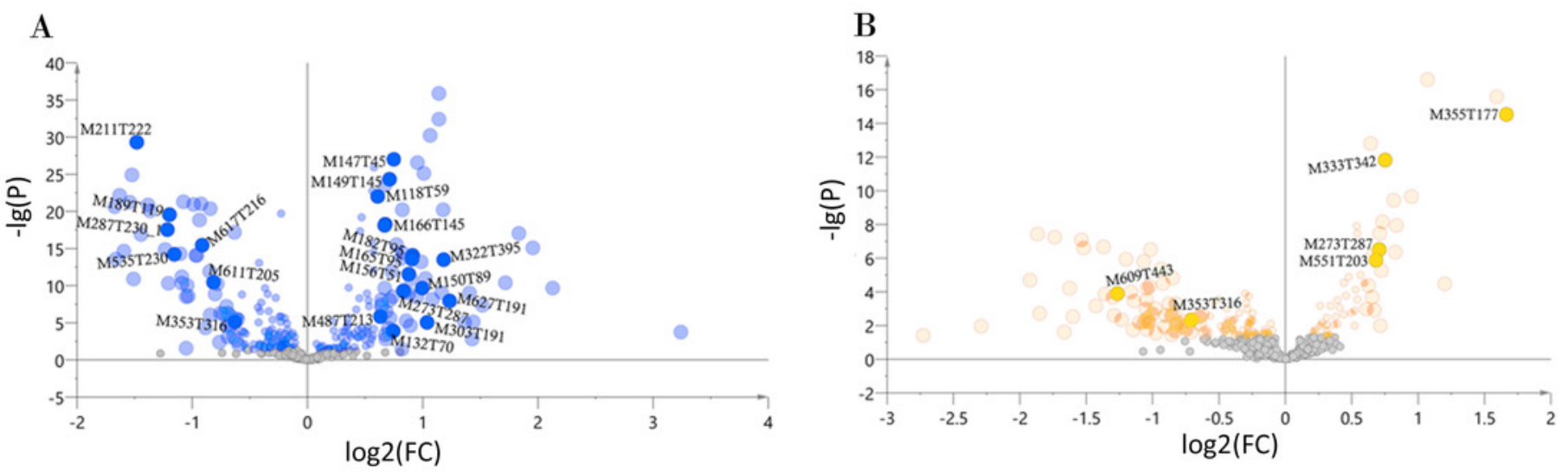

C

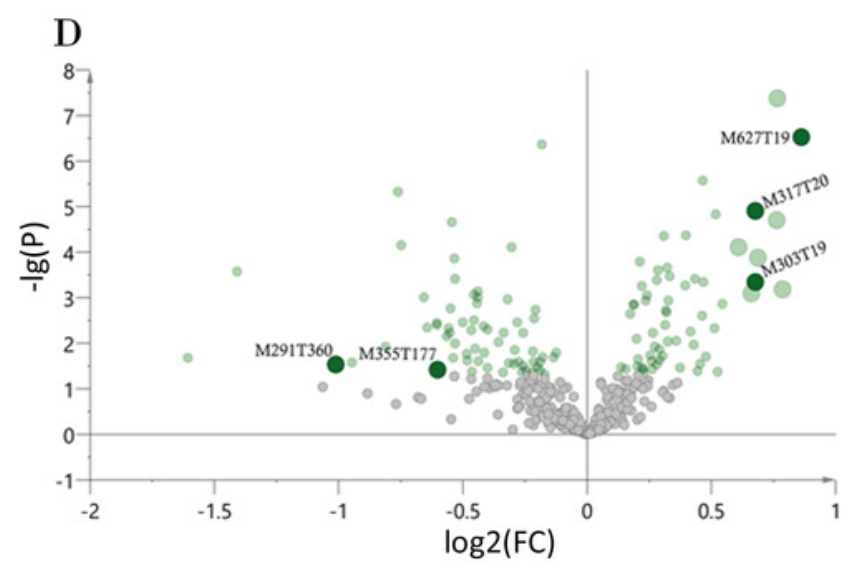


Figure 6

Venn plot of the screened chemical markers in four classified chemotypes
chemotype III
chemotype IV

chemotype I

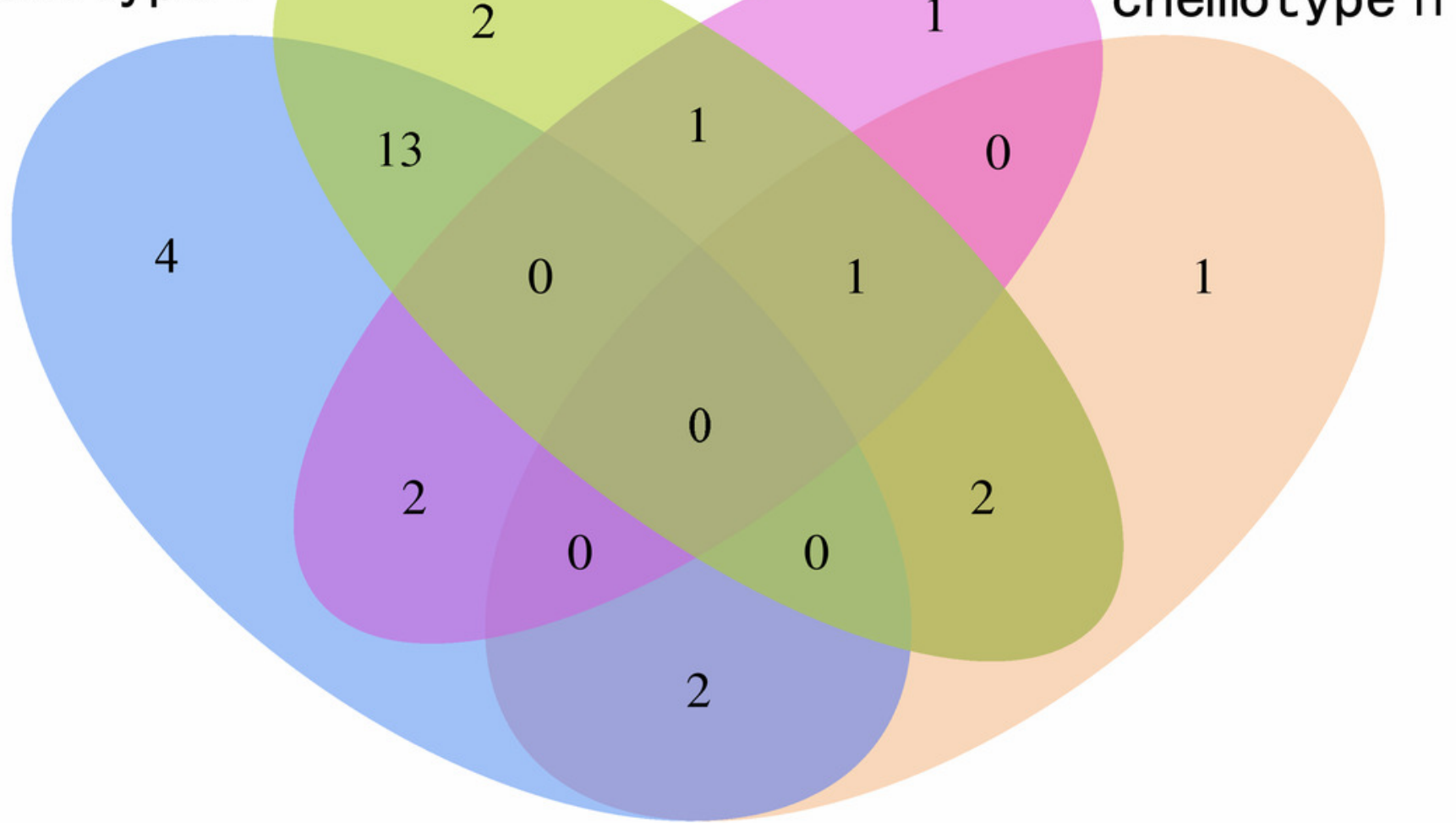


Figure 7

Heat map visualization for discriminating four chemotypes by 29 screened chemical markers

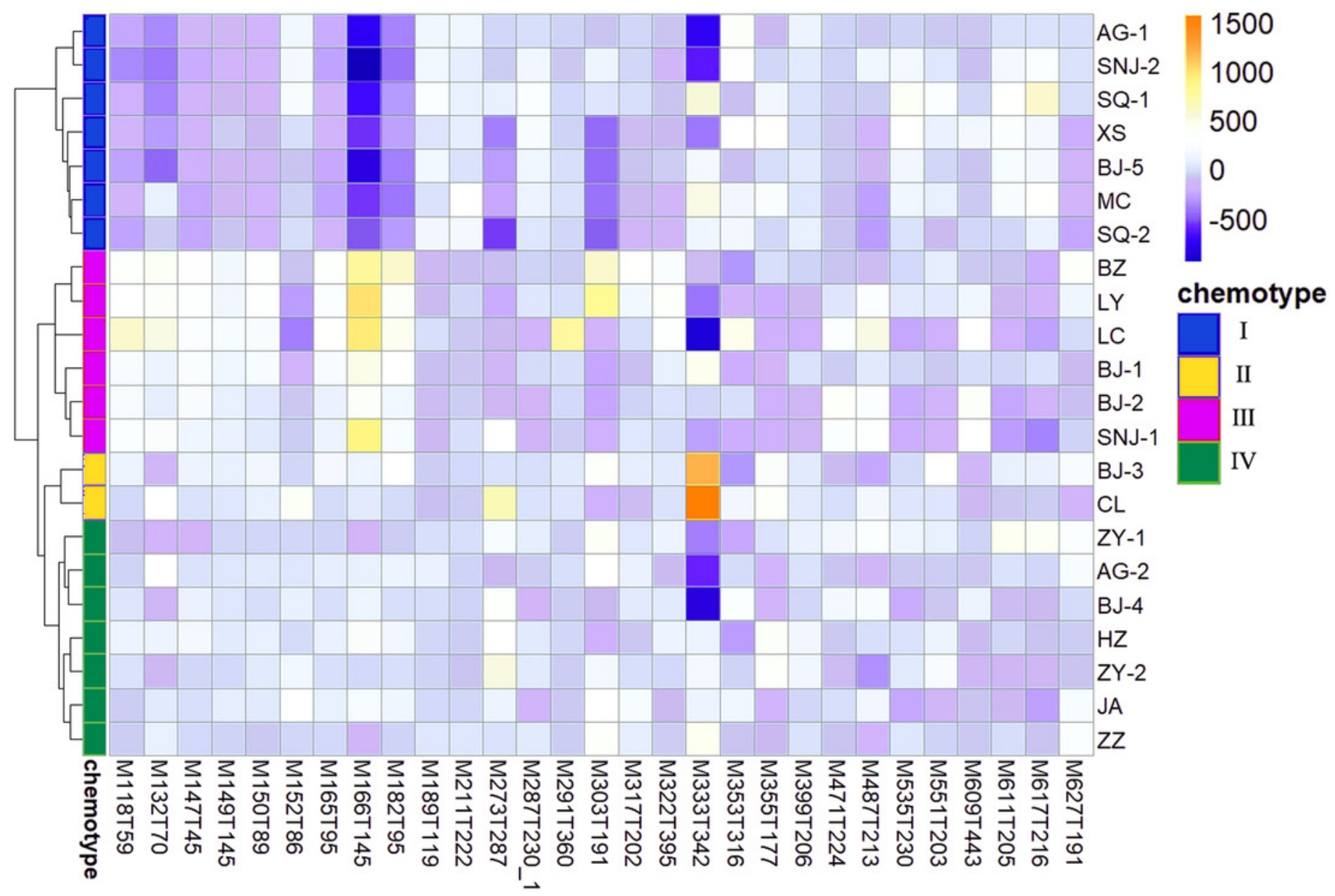


Table $\mathbf{1}$ (on next page)

Statistics of the annotated metabolites 
Table 1. Statistics of the annotated metabolites

\begin{tabular}{cccccc}
\hline Mode & $\begin{array}{c}\text { Number of } \\
\text { all features }\end{array}$ & $\begin{array}{c}\text { Number of } \\
\text { high qulity } \\
\text { features }\end{array}$ & $\begin{array}{c}\text { Number of MS1 } \\
\text { candidate } \\
\text { metabolites }\end{array}$ & $\begin{array}{c}\text { Number of MS1 } \\
\text { annotated } \\
\text { metabolites }\end{array}$ & $\begin{array}{c}\text { Number of MS2 } \\
\text { annotated } \\
\text { metabolites }\end{array}$ \\
\hline ESI+ & 11306 & 9050 & 9686 & 6092 & 451 \\
ESI- & 8616 & 7487 & 6030 & 3554 & 325 \\
\hline
\end{tabular}

2 
Table 2 (on next page)

Main assessment index of each OPLS-DA model 
Table 2. Main assessment index of each OPLS-DA model

\begin{tabular}{ccccccc}
\hline Model & $\begin{array}{c}\text { Orthogonal } \\
\text { Component }\end{array}$ & $\begin{array}{c}\text { Predictive } \\
\text { Component }\end{array}$ & $\begin{array}{c}\mathbf{R}^{2} \mathbf{Y} \\
\mathbf{c u m})\end{array}$ & $\begin{array}{c}\mathbf{Q}^{2} \mathbf{Y}(\mathbf{c u} \\
\mathbf{m})\end{array}$ & $\begin{array}{c}\text { Intercept of } \\
\mathbf{R}^{2}\end{array}$ & $\begin{array}{c}\text { Intercept } \\
\text { of } \mathbf{Q}^{2}\end{array}$ \\
\hline $\begin{array}{c}\text { chemotype I against } \\
\text { the other types }\end{array}$ & 1 & 2 & 0.904 & 0.878 & 0.231 & -0.361 \\
$\begin{array}{c}\text { chemotype II against } \\
\text { the other types }\end{array}$ & 1 & 4 & 0.908 & 0.818 & 0.451 & -0.619 \\
$\begin{array}{c}\text { chemotype III against } \\
\text { the other types } \\
\begin{array}{c}\text { chemotype IVagainst } \\
\text { the other types }\end{array}\end{array}$ & 1 & 3 & 0.914 & 0.861 & 0.326 & -0.424 \\
\hline
\end{tabular}

2 